\title{
Design and Control of Distributed Power-Flow Controller (DPFC)
}

\author{
R.Naveena Bhargavi \\ CVR College of Engineering, Department of EEE, Ibrahimpatan, R.R.District, A.P., India \\ Email: bhargavi.rn5@gmail.com
}

\begin{abstract}
This paper represents a new dimension of the Flexible AC Transmission System (FACTS) called as Distributed Power-Flow Controller (DPFC). The aim of this paper is to develop a new type of power-flow controlling device that offers the same control capability as the UPFC, at a reduced cost and with increased reliability. The new device, the so-called Distributed Power Flow Controller (DPFC), is a further development of the UPFC. The DPFC eliminates the common DC link within the UPFC, to enable the independent operation of the shunt and the series converters which enhances the effective placement of the series and shunt converters.
\end{abstract}

Index Terms-FACTS, Power Flow Controlling Devices, UPFC, DPFC.

\section{INTRODUCTION}

\section{A. Power Flow Controlling Devices}

Power flow is controlled by adjusting the parameters of a system, such as voltage magnitude, line impedance and transmission angle. The device that attempts to vary system parameters to control the power flow can be described as a Power Flow Controlling Device (PFCD)[3].Depending on how devices are connected in systems, PFCDs can be divided into shunt devices, series devices, and combined devices (both in shunt and series with the system), as shown in Figure 1.

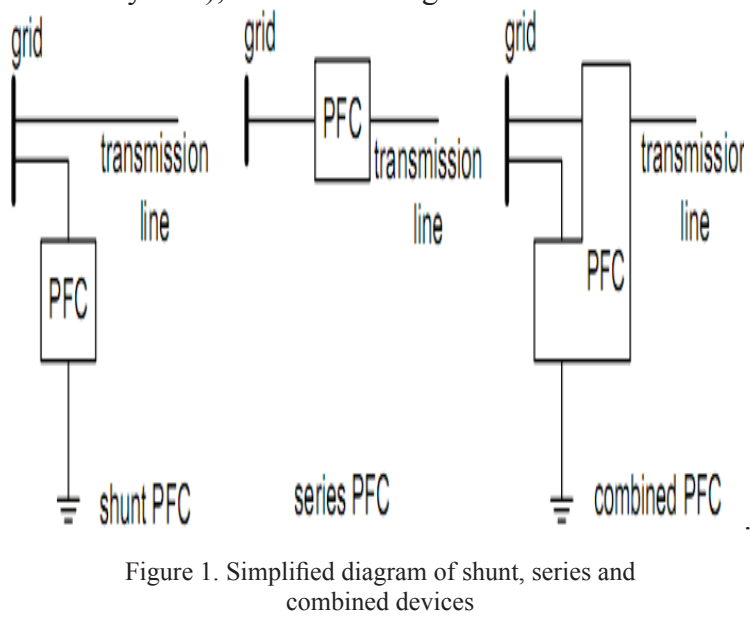

Based on the implemented technology, PFCDs can be categorized into mechanical-based devices and power electronics (PE)-based devices.

Mechanical PFCDs consist of fixed or mechanical interchangeable passive components, such as inductors or capacitors, together with transformers. Typically, mechanical PFCDs have relatively low cost and high reliability. However, because of their relatively low switching speed (from several seconds to minutes) and step-wise adjustments of mechanical PFCDs, they have relatively poor control capability and are not suitable for complex networks of the future.

PE PFCDs also contain passive components, but include additional PE switches to achieve smaller steps and faster adjustments. There is another term - Flexible AC Transmission System (FACTS) - that overlaps with the PE PFCDs. According to the IEEE, FACTS is defined as an 'alternating current transmission system incorporating power electronic based and other static controllers to enhance controllability and increase power transfer capability'. Normally, the High Voltage DC transmission (HVDC) and PE devices that are applied at the distribution network, such as a Dynamic Voltage Restorer (DVR), are also considered as FACTS controllers. Most of the FACTS controllers can be used for power flow control. However, the HVDC and the DVR are out of the scope of the PFCD. The relationship between the PFCDs, FACTS controllers and mechanical controller is shown in Figure 2.

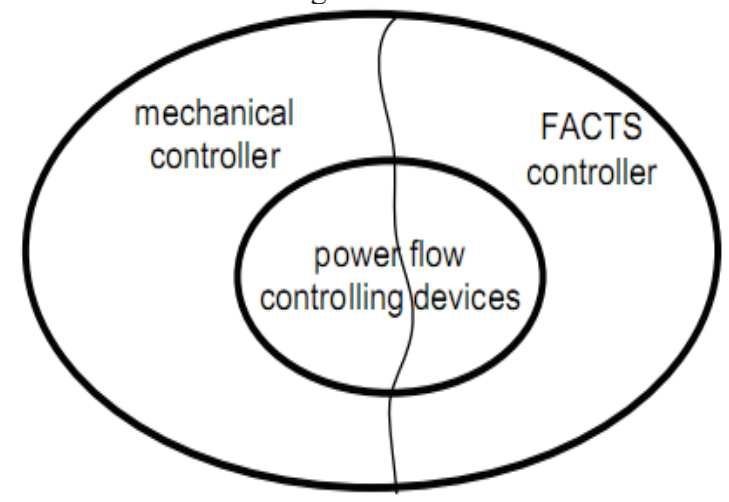

Figure 2. Relationship between the PFCDs, FACTS controllers and mechanical controller

PE PFCD devices can be further subcategorized into two types according to the applied switch technologies: thyristor-based devices and Voltage Source Converter (VSC) based devices. Thyristor PFCDs use inverseparallel thyristors in series or in parallel with passive components. By controlling the firing angle of the thyristors, the impedance of the device can be adjusted. A thyristor can be controlled to turn on but not to turn off.

It will turn off automatically when the current goes negative. Consequently, the thyristor can only be turned on once within one cycle. The switching frequency of thyristor PFCDs is therefore limited to the system frequency $(50 / 60 \mathrm{~Hz})$, resulting in low switching losses. Because thyristors can handle larger voltages and currents 
than other power semiconductors, the power level of thyristor PFCDs are also higher. The thyristor PFCDs are simpler than VSC PFCDs, allowing them higher reliability. However, the waveforms of voltages and currents generated by thyristor PFCDs contain a large amount of harmonics, thereby requiring large filters.

VSC PFCDs employ advanced switch technologies, such as Insulated Gate Bipolar Transistors (IGBT), Insulated Gate Commutated Thyristors (IGCT), or Metal Oxide Semiconductor Field Effect Transistors (MOSFET) to build converters. Because these switches have turn-on and turn-off capability, the output voltage of a VSC is independent from the current. Consequently, it is possible to turn the switches on and off within the VSC multiple times within one cycle. Several types of VSCs have been developed, such as multi-pulse converters, multi-level converters, square-wave converters, etc. These VSCs proved a free controllable voltage in both magnitude and phase. Due to their relatively high switching frequency, VSC PFCDs make practically instant control (less than one cycle) possible. High switching frequencies also reduce low frequency harmonics of the outputs and even enable PFCDs to compensate disturbances from networks. Therefore, VSC PFCDs are the most suitable devices for future power systems.

On the other hand, there are some challenges facing VSC PFCDs. Firstly, because large amounts of switches are connected in series or in parallel to allow the high voltage and high current through, the VSC PFCDs are expensive. In addition, due to their higher switching frequency and higher on-state voltage in comparison with thyristors, VSC PFCD losses are higher as well. However, with developments in power electronics (such as Silicon-carbide, Gallium-Nitride and synthetic diamond), VSC PFCDs can become more feasible and cost-effective in the future.

According to the above considerations of different types of PFCDs, it can be concluded that PE combined PFCDs (also referred to as combined FACTS) have the best control capability among all PFCDs. They

inherit the advantages of PE PFCDs and combined PFCDs, which is the fast adjustment of multiple system parameters. The Unified Power Flow Controller (UPFC) and Interline Power Flow Controller (IPFC) are currently the most powerful PFCDs; they can adjust all system parameters: line impedance, transmission angle, and bus voltage.

\section{UPFC AND IPFC}

\section{A. UPFC (Unified Power Flow Controller)}

The Unified Power Flow Controller is comprised of a STATCOM and a SSSC coupled via a Common DC link to allow bi-directional flow of active power between the series output terminals of the SSSC and the shunt output terminals of the STATCOM. Each converter can independently generate (or) absorb reactive power at its own AC terminal. The two converters are operated from a DC link provided by a DC storage capacitor. The configuration of a UPFC is shown in Figure 3.

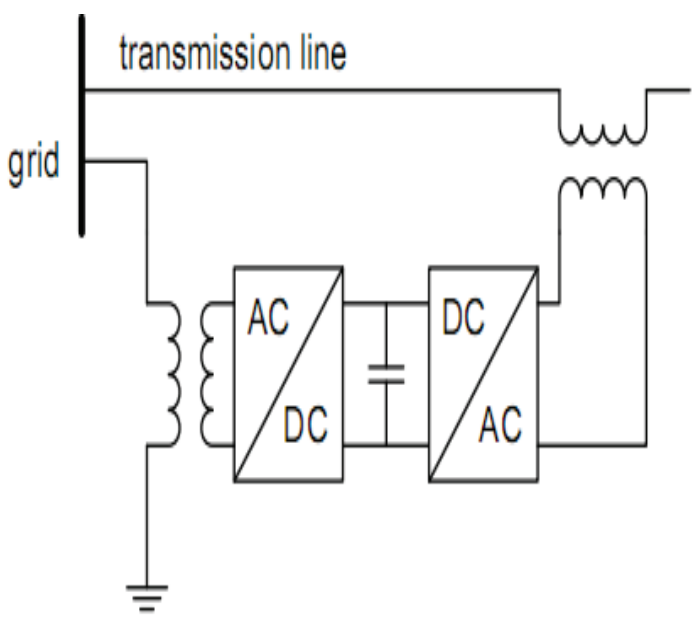

Figure 3. UPFC Configuration

The series converter executes the main function of the UPFC by injecting a voltage, with controllable magnitude and phase angle, in series with the transmission line. It is controlled to provide concurrent active and reactive series compensation without an external energy source. By means of the series voltage injection without angular constraint, the UPFC is able to control, concurrently or selectively, the transmission angle, impedance and line voltage or, alternatively, active and reactive power flow through the line. The link voltage injected by the series converter results in active and reactive power exchange between the series converter and the transmission line. The reactive power is generated internally by the series converter (like SSSC), and the active power is supplied by the shunt converter that is transported through the common DC. The basic function of the shunt converter is to supply or absorb the active power demanded by the series converter. The shunt converter controls the voltage of the DC capacitor by absorbing or generating active power from the bus, therefore it acts as a synchronous source in parallel with the system. Similar to the STATCOM, the shunt converter can also provide independently controllable reactive compensation for the bus.

B.IPFC (Interline Power Flow Controller)

The Interline Power Flow Controller (IPFC) consists of the two (or more) series converters in different transmission lines that are inter-connected via a common DC link, as shown in Figure 4. Unlike other FACTS devices that aim to control the parameter of a single transmission line, the IPFC is conceived for the compensation and control of power flow in a multi-line transmission system [4].

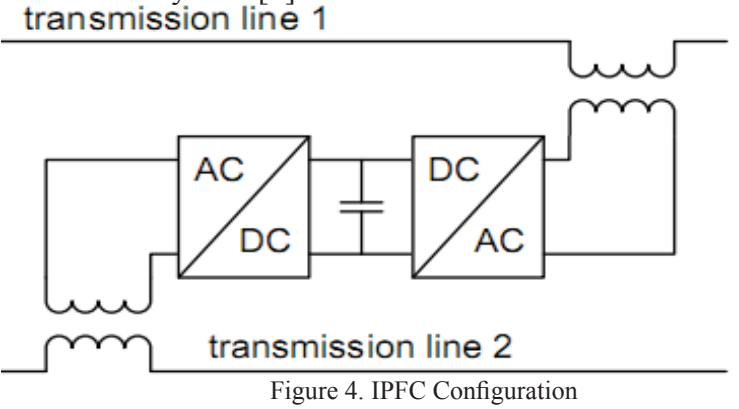


Each converter can provide series reactive compensation of its own line, just as an SSSC can. As the converters can exchange active power through their common DC link, the IPFC can also provide active compensation. This allows the IPFC to provide both active and reactive compensation for some of the lines and thereby optimize the utilization of the overall transmission system. Note that the active power supplied to one line is taken from the other lines. If required, the IPFC can be complemented with an additional shunt converter to provide active power from a suitable shunt bus.

However, the UPFC and IPFC are not widely applied in practice, due to their high cost and the susceptibility to failures. Generally, the reliability can be improved by reducing the number of components; however, this is not possible due to the complex topology of the UPFC and IPFC.

\section{Distributed Power Flow Controller(DPFC)}

To reduce the failure rate of the components by selecting components with higher ratings than necessary or employing redundancy at the component or system levels are also options. Unfortunately, these solutions increase the initial investment necessary, negating any cost-related advantages. Accordingly, new approaches are needed in order to increase reliability and reduce cost of the UPFC and IPFC at the same time.

The elimination of the common DC link also allows the DSSC concept to be applied to series converters. In that case, the reliability of the new device is further improved due to the redundancy provided by the distributed series converters.

Unlike in UPFC where the active power transfer is through the DC link between the series and shunt converters [7] here in DPFC this power flow is through the transmission lines at the third harmonic frequency which is a zero-sequence component and can be naturally blocked by a Y- $\Delta$ transformer. The DPFC makes use of the distributed FACTS (D-FACTS) in the design of the series converter, which is to use multiple single-phase converters instead of one large rated three phase converter while the shunt converter remains as static synchronous compensator (STATCOM) as in UPFC. These large numbers of series converters provides redundancy, thereby increasing the system reliability. As the D-FACTS converters are single-phase and floating with respect to the ground, there is no high-voltage isolation required between the phases. Accordingly, the cost of the DPFC system is lower than the UPFC. The controllability of the DPFC is same as that of the UPFC which refers to the adjustment of the line impedance, the transmission angle, and the bus voltage. The operation principle, the modeling and control, and experimental demonstrations of the DPFC are presented in this paper and the corresponding laboratory results that are carried out on a scaled prototype are also shown.

IV.DPFC OPERATING PRINCIPLE AND CONTROL A.DPFC Operating Principle

Within the DPFC, the transmission line presents a common connection between the $\mathrm{AC}$ ports of the shunt and the series converters. Therefore, it is possible to exchange active power through the AC ports. The method is based on power theory of non-sinusoidal components.

According to the Fourier Analysis, non- sinusoidal voltage and current be expressed as the sum of sinusoidal functions in different frequencies with different amplitudes. The active power resulting from this nonsinusoidal voltage and current is defined as the mean value of the product of voltage and current. Since the integrals of all the cross product of terms with different frequencies are zero, the active power can be expressed $P=\sum_{i=1}^{\infty} V_{i} I_{i} \cos \phi_{i}$

Where $V_{i}$ and $I_{i}$ are the voltage and current at the $i^{\text {th }}$ harmonic frequency respectively, and $\varphi_{i}$ is the corresponding angle between the voltage and current. Equation (3.1) shows that the active powers at different frequencies are independent from each other and the voltage or current at one frequency has no influence on the active power at other frequencies. The independence of the active power at different frequencies gives the possibility that a converter without a power source can generate active power at one frequency and absorb this power from other frequencies.

By applying this method to the DPFC, the shunt converter can absorb active power from the grid at the fundamental frequency and inject the power back at a harmonic frequency. This harmonic active power flows through a transmission line equipped with series converters. According to the amount of required active power at the fundamental frequency, the DPFC series converters generate a voltage at the harmonic frequency, thereby absorbing the active power from harmonic components. Neglecting losses, the active power generated at the fundamental frequency is equal to the power absorbed at the harmonic frequency. For a better understanding, Figure 5 indicates how the active power is exchanged between the shunt and the series converters in the DPFC system.

The high-pass filter within the DPFC blocks the fundamental frequency components and allows the harmonic components to pass, thereby providing a return path for the harmonic components. The shunt and series converters, the high pass filter and the ground form a closed loop for the harmonic current.

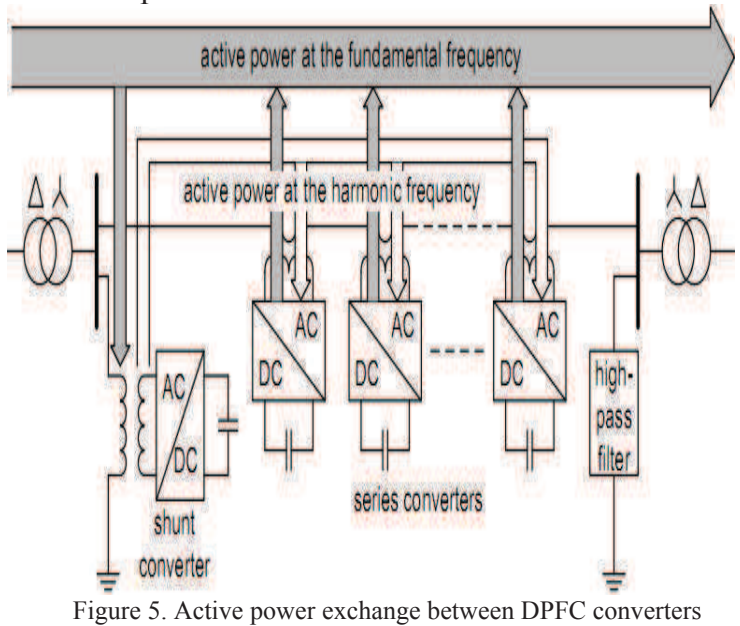




\section{B.DPFC Control}

To control multiple converters, a DPFC consists of three types of controllers: central control, shunt control and series control, as shown in Figure 5.

The shunt and series control are localized controllers and are responsible for maintaining their own converters' parameters. The central control takes care of the DPFC functions at the power system level. The function of each controller is listed below.

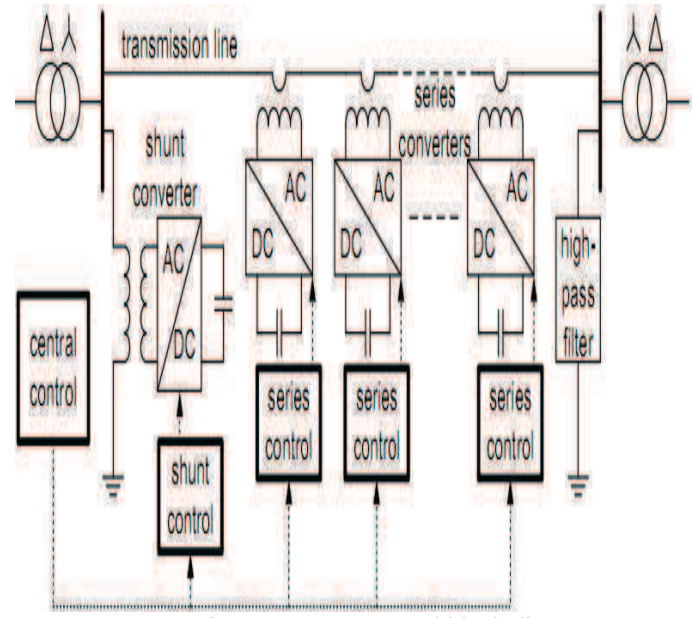

Figure 5. DPFC control block diagram

(a) Central control: The central control generates the reference signals for both the shunt and series converters of the DPFC. Its control function depends on the specifics of the DPFC application at the power system level, such as power flow control, low frequency power oscillation damping and balancing of asymmetrical components. According to the system requirements, the central control gives corresponding voltage reference signals for the series converters and reactive current signal for the shunt converter. All the reference signals generated by the central control concern the fundamental frequency components.

(b) Series control: Each series converter has its own series control. The controller is used to maintain the capacitor DC voltage of its own converter, by using $3^{\text {rd }}$ harmonic frequency components, in addition to generating series voltage at the fundamental frequency as required by the central control.

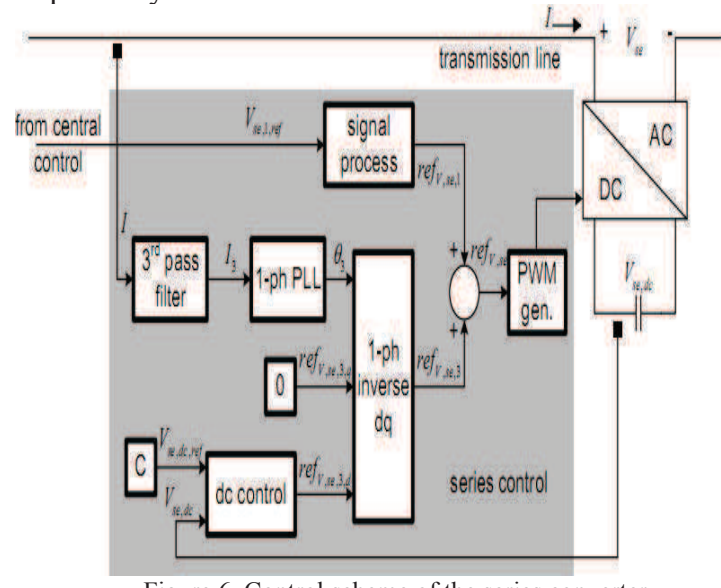

Figure 6. Control scheme of the series converter (c) Shunt control: The objective of the shunt control is to inject a constant $3^{\text {rd }}$ harmonic current into the line to supply active power for the series converters. At the same time, it maintains the capacitor DC voltage of the shunt converter at a constant value by absorbing active power from the grid at the fundamental frequency and injecting the required reactive current at the fundamental frequency into the grid.

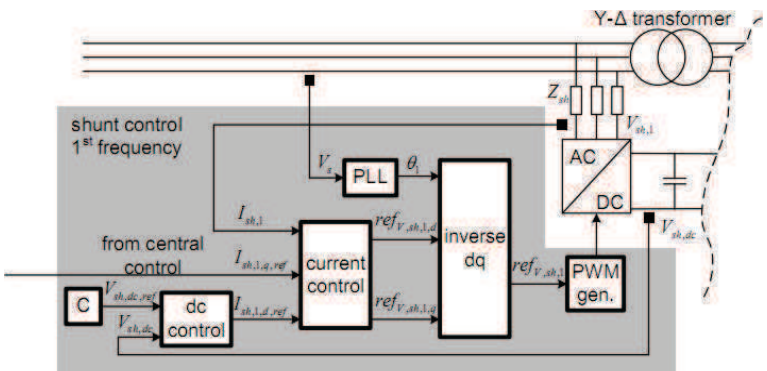

(a)

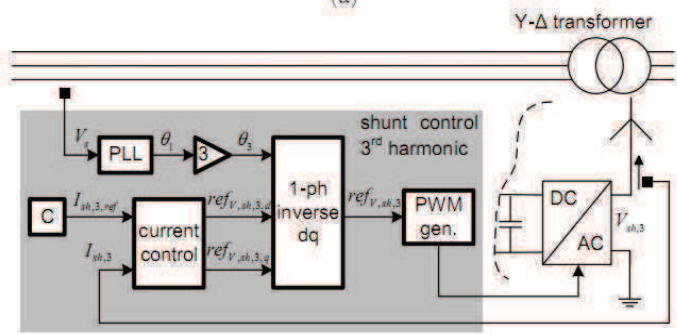

(b)

Figure 7. Control scheme of the shunt converter: (a) for the fundamental frequency components (b) for the 3rd harmonic frequency components

\section{Simulation RESUlTS}

The DPFC model is developed in the MATLAB Simulink environment. The main layout and simulation results are shown.

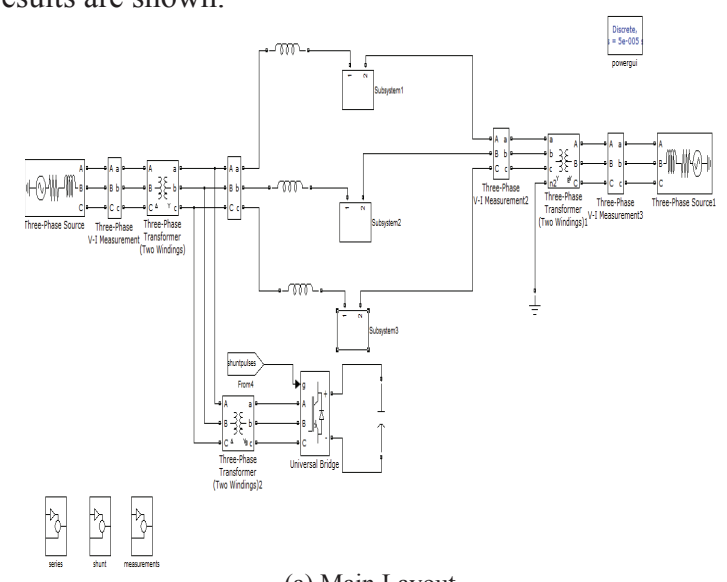

(a) Main Layout

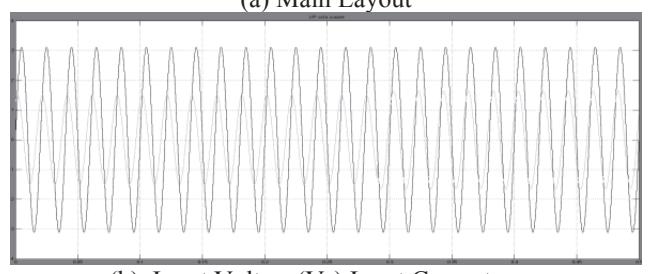

(b) Input Voltage(Vs) Input Current 


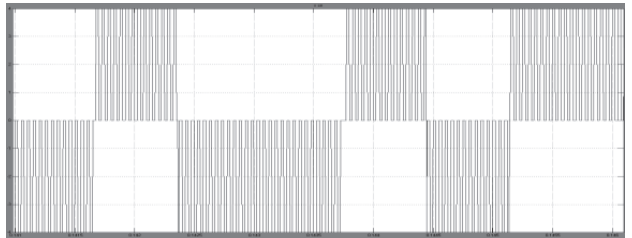

(c) Series Converter Output

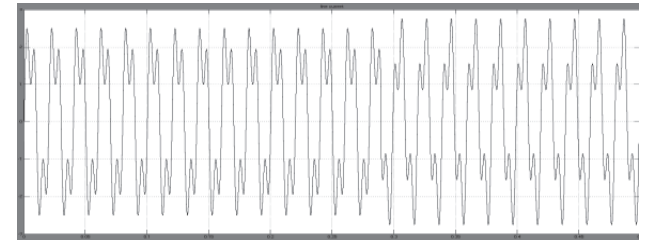

(d) Line Current

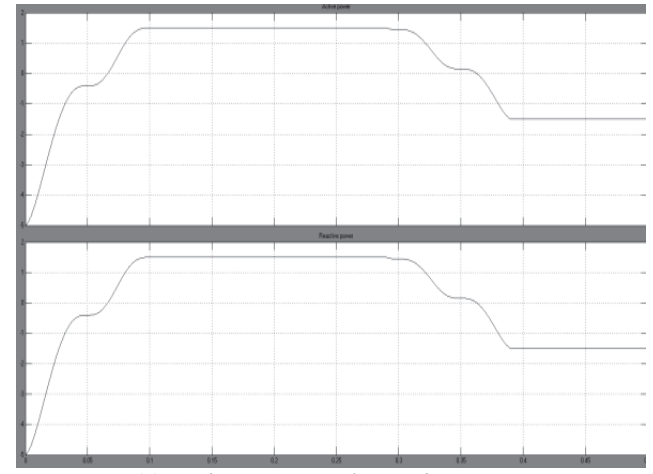

(e) Active Power and Reactive Power

\section{CONCLUSIONS}

The DPFC basic control is developed based on the dynamic model. The basic control stabilizes the level of the capacitor DC voltage of each converter and ensures that the converters inject the voltages into the network according to the command from the central control. The shunt converter injects a constant current at the $3 \mathrm{rd}$ harmonic frequency, while its DC voltage is stabilized by the fundamental frequency component. For the series converter, the reference of the output voltage at the fundamental frequency is obtained from the central control and the DC voltage level is maintained by the $3 \mathrm{rd}$ harmonic components. The control parameters of the basic control are determined. Both the model and the basic control are verified in Matlab Simulink.

Utilizing the DPFC to damp low-frequency power oscillation is investigated. The DPFC is used to damp the inter-area oscillatory modes. Because the DPFC can simultaneously adjust three system parameters, namely the bus voltage, the line impedance and the transmission angle, a maximum of three POD controllers can be applied to one DPFC. Within the thesis, the POD controller is designed using the residue method and a two-area network is used in the case study. From the simulation, it can be seen that the DPFC can shift three critical oscillatory modes at the same time. Therefore, it can be concluded that the DPFC has the same capability as the UPFC for power oscillation damping.

Employing the DPFC for asymmetry compensation is studied. Because of the active power exchange between the shunt and the series converters, the DPFC can compensate both active and reactive asymmetry at the fundamental frequency. In addition, since the series converter is single-phase converter, the DPFC can compensate for both zero and negative sequence components. Accordingly, the DPFC currently is the most versatile device for asymmetry compensation among all FACTS devices.

\section{REFERENCES}

[1] L. Gyugyi, C. D. Schauder, S. L. Williams, T. R. Rietman, D. R. Torgerson, and A. Edris. "The unified power flow controller: a new approach to power transmission control". Power Delivery, IEEE Transactions on, 1995.

[2] L. Gyugyi, K. K. Sen, and C. D. Schauder. "The interline power flow con-troller concept: a new approach to power flow management in transmission systems". Power Delivery, IEEE Transactions on, 1999.

[3] D. Van Hertem, J. Verboomen, R. Belmans, and W. L. Kling. "Power flow controlling devices: an overview of their working principles and their applica-tion range". In: Future Power Systems, International Conference on, 2005.

[4] R. L. Vasquez Arnez. "The Interline Power Flow Controller: Further aspects related to its operation and main limitations". In: Transmission andDistribution Conference and Exposition, IEEE/PES, 2008.

[5] Yuan, Z.; de Haan, S.W.H.; Ferreira, B.: "UPFC with Eliminated Common DCLink Connection between Shunt and Series Part", IEEE Power Engineering Society General Meeting (PESGM) 2007, Tampa, USA.

[6] Yuan, Z.; de Haan, S.W.H.; Ferreira, B.: "A New FACTS Component - Distributed Power Flow Controller (DPFC)", European Conference on Power Electronics Applications (EPE) 2007, Aalborg, Denmark.

[7] Yuan, Z.; de Haan, S.W.H.; Ferreira, B.: "A Method of Transmitting Active Power Between Converters with Eliminated Common DC Link", IEEE Benelux Young Research Symposium in Electrical Power Engineering 2008, Eindhoven, the Netherlands. 